\title{
Proofs of some conjectures on monotonicity of ratios of Kummer, Gauss and generalized hypergeometric functions.
}

\author{
Khaled Mehrez, Sergei M. Sitnik
}

\begin{abstract}
In the preprint [1] one of the authors formulated some conjectures on monotonicity of ratios for exponential series sections. They lead to more general conjecture on monotonicity of ratios of Kummer hypergeometric functions and was not proved from 1993. In this paper we prove some conjectures from [1] for Kummer hypergeometric functions and its further generalizations for Gauss and generalized hypergeometric functions. The results are also closely connected with Turán-type inequalities.
\end{abstract}

\section{Introduction and statement of problems.}

Let us consider the series for the exponential function

$$
\exp (x)=e^{x}=\sum_{k=0}^{\infty} \frac{x^{k}}{k !}, x \geq 0,
$$

its section $S_{n}(x)$ and series remainder $R_{n}(x)$ in the form

$$
S_{n}(x)=\sum_{k=0}^{n} \frac{x^{k}}{k !}, R_{n}(x)=\exp (x)-S_{n}(x)=\sum_{k=n+1}^{\infty} \frac{x^{k}}{k !}, x \geq 0 .
$$

Besides simplicity and elementary nature of these functions many mathematicians studied problems for them. G. Szegó proved a remarkable limit distribution for zeroes of sections, accumulated along so-called the Szegó curve ([2]). S. Ramanujan seems was the first who proved the non-trivial inequality for exponential sections in the form ([3], pp. 323-324) : if

$$
\frac{e^{n}}{2}=R_{n-1}(n)+\frac{n^{n}}{n !} \theta(n)
$$

then

$$
\frac{1}{3}<\theta(n)=\frac{n !\left(\frac{e^{n}}{2}-R_{n-1}(n)\right)}{n^{n}}<\frac{1}{2} .
$$

This result is important as it also evaluates $e^{n}$ in rational bounds

$$
\frac{2 n^{n}}{3 n !}+2 R_{n-1}(n)<e^{n}<\frac{n^{n}}{n !}+2 R_{n-1}(n)
$$

as it was specially pointed out in ([3], pp. 323-324). 
In the preprint 1] were thoroughly studied inequalities of the form

$$
m(n) \leq f_{n}(x)=\frac{R_{n-1}(x) R_{n+1}(x)}{\left[R_{n}(x)\right]^{2}} \leq M(n), x \geq 0 .
$$

The search for the best constants $m(n)=m_{\text {best }}(n), M(n)=M_{\text {best }}(n)$ has some history. The left-hand side of (2) was first proved by Kesava Menon in [4] with $m(n)=\frac{1}{2}$ (not best) and by Horst Alzer in [5] with

$$
m_{\text {best }}(n)=\frac{n+1}{n+2}=f_{n}(0),
$$

cf. [1] for the more detailed history. In [1] it was also shown that in fact the inequality (2) with the sharp lower constant (3) is a special case of the stronger inequality proved earlier in 1982 by Walter Gautschi in 6 .

It seems that the right-hand side of (2) was first proved by the author in [1] with $M_{\text {best }}=1=f_{n}(\infty)$. In [1] dozens of generalizations of inequality (2) and related results were proved. May be in fact it was the first example of so called Turan-type inequality for special case of the Kummer hypergeometric functions, recently this class of inequalities became thoroughly studied (cf. [10]-[18]).

Obviously the above inequalities are consequences of the next conjecture originally formulated in [1] and recently revived in [8]-9].

Conjecture 1. The function $f_{n}(x)$ in (2) is monotone increasing for $x \in[0 ; \infty), n \in \mathbb{N}$. So the next inequality is valid

$$
\frac{n+1}{n+2}=f_{n}(0) \leq f_{n}(x)<1=f_{n}(\infty)
$$

In 1990's we tried to prove this conjecture in the straightforward manner by expanding an inequality $\left(f_{n}(x)\right)^{\prime} \geq 0$ in series and multiplying triple products of hypergeometric functions but failed ([7] -8 ).

Consider a representation via Kummer hypergeometric functions

$$
f_{n}(x)=\frac{n+1}{n+2} g_{n}(x), g_{n}(x)=\frac{{ }_{1} F_{1}(1 ; n+1 ; x){ }_{1} F_{1}(1 ; n+3 ; x)}{\left[{ }_{1} F_{1}(1 ; n+2 ; x)\right]^{2}} .
$$

So the conjecture 1 may be reformulated in terms of this function $g_{n}(x)$ as conjecture 2 .

Conjecture 2. The function $g_{n}(x)$ in (5) is monotone increasing for $x \in[0 ; \infty), n \in \mathbb{N}$.

This leads us to the next more general

Problem 1. Find monotonicity in $x$ conditions for $x \in[0 ; \infty)$ for all parameters $a, b, c$ for the function

$$
h(a, b, c, x)=\frac{{ }_{1} F_{1}(a ; b-c ; x){ }_{1} F_{1}(a ; b+c ; x)}{\left[{ }_{1} F_{1}(a ; b ; x)\right]^{2}} .
$$

We may also call (6) mockingly (in Ramanujan way, remember his mock theta-functions!) "The abc-problem" for Kummer hypergeometric functions, why not?

Another generalization is to change Kummer hypergeometric functions to higher ones. 
Problem 2. Find monotonicity in $x$ conditions for $x \in[0 ; \infty)$ for all vector-valued parameters a,b,c for the function

$$
\begin{aligned}
& h_{p, q}(a, b, c, x)=\frac{{ }_{p} F_{q}(a ; b-c ; x){ }_{p} F_{q}(a ; b+c ; x)}{\left[{ }_{p} F_{q}(a ; b ; x)\right]^{2}}, \\
& a=\left(a_{1}, \ldots, a_{p}\right), b=\left(b_{1}, \ldots, b_{q}\right), c=\left(c_{1}, \ldots, c_{q}\right) .
\end{aligned}
$$

This is "The abc-problem" for generalized hypergeometric functions. The more complicated problems are obvious and may be considered for pairs or triplets of parameters and also for multivariable hypergeometric functions.

The aim of this paper is to prove conjectures 1 and 2, and to find conditions for validity of problems 1 and 2 and so completely solve them, cf. also [28].

\section{Two lemmas}

We formulate two useful lemmas which will be used below. These lemmas were first proved in $([23])$, cf. also $([24])-([25])$ for the detailed proof and further applications.

Lemma 1 Let $\left(a_{n}\right)$ and $\left(b_{n}\right)(n=0,1,2 \ldots)$ be real numbers, such that $b_{n}>$ $0, n=0,1,2, \ldots$ and $\left(\frac{a_{n}}{b_{n}}\right)_{n \geq 0}$ is increasing (decreasing), then $\left(\frac{a_{0+\ldots}+a_{n}}{b_{0}+\ldots+b_{n}}\right)_{n}$ is increasing (decreasing).

Lemma 2 . Let $\left(a_{n}\right)$ and $\left(b_{n}\right)(n=0,1,2 \ldots)$ be real numbers and let the power series $A(x)=\sum_{n=0}^{\infty} a_{n} x^{n}$ and $B(x)=\sum_{n=0}^{\infty} b_{n} x^{n}$ be convergent if $|x|<r$. If $b_{n}>0, n=0,1,2, \ldots$ and if the sequence $\left(\frac{a_{n}}{b_{n}}\right)_{n \geq 0}$ is (strictly) increasing (decreasing), then the function $\frac{A(x)}{B(x)}$ is also (strictly) increasing on $[0, r[$.

\section{Monotonicity for the Kummer hypergeometric function and associated Turán type inequality}

Theorem 1 Let $a, b, c$ be real numbers such that $0<a<b-c$ and $b>1$ and the function $x \longmapsto h(a, b, c, x)$ is defined by

$$
h(a, b, c, x)=\frac{{ }_{1} F_{1}(a ; b-c ; x){ }_{1} F_{1}(a ; b+c ; x)}{\left[{ }_{1} F_{1}(a ; b ; x)\right]^{2}} .
$$

Then this function is increasing on $[0, \infty[$. Consequently, for $n \in \mathbb{N}$, the functions $x \longmapsto f_{n}(x)$ in (2) and $x \longmapsto g_{n}(x)$ in (5) are also increasing on $[0, \infty[$.

Proof For all $a, b, c$ be real numbers such that $0<a<b-c$ and $b>1$ we evualate

$$
\begin{aligned}
h(a, b, c, x) & =\frac{{ }_{1} F_{1}(a ; b-c ; x)_{1} F_{1}(a ; b+c ; x)}{\left[{ }_{1} F_{1}(a ; b ; x)\right]^{2}}= \\
& =\frac{\left(\sum_{n=0}^{\infty} \frac{(a)_{n}}{(b-c)_{n} n !} x^{n}\right)\left(\sum_{n=0}^{\infty} \frac{(a)_{n}}{(b+c)_{n} n !} x^{n}\right)}{\left[\sum_{n=0}^{\infty} \frac{(a)_{n}}{(b)_{n} n !} x^{n}\right]^{2}}= \\
& =\frac{\sum_{n=0}^{\infty} A_{n} x^{n}}{\sum_{n=0}^{\infty} B_{n} x^{n}}
\end{aligned}
$$


where

$$
A_{n}=\sum_{k=0}^{n} \frac{(a)_{k}(a)_{n-k}}{(b-c)_{k}(b+c)_{n-k} k !(n-k) !} \text { and } B_{n}=\sum_{k=0}^{n} \frac{(a)_{k}(a)_{n-k}}{(b)_{k}(b)_{n-k} k !(n-k) !} \text {. }
$$

Let define sequences $\left(u_{n, k}\right)_{k \geq 0},\left(v_{n, k}\right)_{k \geq 0}$ and $\left(w_{n, k}\right)_{k \geq 0}$ by

$$
u_{n, k}=\frac{(a)_{k}(a)_{n-k}}{(b-c)_{k}(b+c)_{n-k} k !(n-k) !}, \quad v_{n, k}=\frac{(a)_{k}(a)_{n-k}}{(b)_{k}(b)_{n-k} k !(n-k) !},
$$

and

$$
w_{n, k}=\frac{u_{n, k}}{v_{n, k}}=\frac{(b)_{k}(b)_{n-k}}{(b-c)_{k}(b+c)_{n-k}}, k \geq 0 .
$$

It follows that

$$
\begin{aligned}
\frac{w_{n, k+1}}{w_{n, k}} & =\frac{u_{n, k+1} v_{n, k}}{v_{n, k+1} u_{n, k}}= \\
& =\frac{(b)_{k+1}(b)_{n-k-1}(b-c)_{k}(b+c)_{n-k}}{(b-c)_{k+1}(b+c)_{n-k-1}(b)_{k}(b)_{n-k}}= \\
& =\frac{\Gamma(b+k+1)}{\Gamma(b+k)} \cdot \frac{\Gamma(b+n-k-1)}{\Gamma(b+n-k)} \cdot \frac{\Gamma(b-c+k)}{\Gamma(b-c+k+1)} \cdot \frac{\Gamma(b+c+n-k)}{\Gamma(b+c+n-k-1)}= \\
& =\frac{(b+k)}{(b-c+k)} \cdot \frac{(b+c+n-k-1)}{(b+n-k-1)} \geq 1 .
\end{aligned}
$$

We conclude that the sequence $\left(w_{n, k}\right)_{k \geq 0}$ is increasing and consequently the sequence $\left(C_{n}=\frac{A_{n}}{B_{n}}\right)_{n \geq 0}$ is also increasing by lemma 1. Thus the function $h(a, b, c, x)$ is increasing on $[0, \infty[$ by lemma 2 . Finally, replacing $a$ and $c$ by 1 and $b$ by $n+1$ for all $n \in \mathbb{N}$, we obtain that the functions $x \longmapsto g_{n}(x)$ and $x \longmapsto f_{n}(x)$ are also increasing on $[0, \infty[$. So both conjectures 1 and 2 from introduction are proved. And also we found the solution to the Problem 1 from introduction if restrictions of the theorem 1 are valid.

Corollary 1 For all $a, b, c$ be real numbers such that $0<a<b-c$ and $b>1$, the following Turán type inequality

$$
\left[{ }_{1} F_{1}(a, b, x)\right]^{2} \leq{ }_{1} F_{1}(a, b-c, x) \cdot{ }_{1} F_{1}(a, b+c, x)
$$

holds for all $x \in[0, \infty[$.

Proof Since the function $x \longmapsto h(a, b, c, x)$ is increasing on $[0, \infty[$, we have

$$
h(a, b, c, x) \geq h(a, b, c, 0)=1 .
$$

This result is interesting as a corollary of monotonicity property we consider, this inequality itself is not new and may be found in ([17]). And in general Turán type inequalities always can be generalized to stronger results on monotonicity of function ratios with unit upper or lower constants. 


\section{Monotonicity for the hypergeometric function and associated Turán type inequality}

Now we also solve the Problem 2 for general hypergeometric-type functions under some natural conditions.

Theorem 2 Let $p, q \in \mathbb{N}$ be such that $p \leq q+1, a=\left(a_{1}, \ldots, a_{p}\right), b=\left(b_{1}, \ldots, b_{q}\right)$, $c=\left(c_{1}, \ldots, c_{q}\right), b_{i}>0, b_{i}-c_{i}>0$ for $i=1,2, \ldots, q$ and $a_{i}>b_{i}$ for $i=2, \ldots, p$. If $b_{i}>1$ for $i=1,2, \ldots, q$, then the function $x \longmapsto h_{p, q}(a, b, c, x)$ in (耳) is strictly increasing on $[0,1[$.

\section{Proof}

By using the power-series representations of the function ${ }_{p} F_{q}(a ; b ; x)$ we have

$$
\begin{aligned}
h_{p, q}(a ; b ; c ; x) & =\frac{{ }_{p} F_{q}(a ; b-c ; x) \cdot{ }_{p} F_{q}(a ; b+c ; x)}{\left({ }_{p} F_{q}(a ; b ; x)\right)^{2}}= \\
& =\frac{\left[\sum_{n=0}^{\infty} \frac{\left(a_{1}\right)_{n}\left(a_{2}\right)_{n} \ldots\left(a_{p}\right)_{n} x^{n}}{\left(b_{1}-c_{1}\right)_{n}\left(b_{2}-c_{2}\right)_{n} \ldots\left(b_{q}-c_{q}\right)_{n} n !}\right]}{\left[\sum_{n=0}^{\infty} \frac{\left(a_{1}\right)_{n}\left(a_{2}\right)_{n} \ldots\left(a_{p}\right)_{n} x^{n}}{\left(b_{1}\right)_{n}\left(b_{2}\right)_{n} \ldots\left(b_{q}\right)_{n} n !}\right]^{2}} . \\
& \cdot\left[\sum_{n=0}^{\infty} \frac{\left(a_{1}\right)_{n}\left(a_{2}\right)_{n} \ldots\left(a_{p}\right)_{n} x^{n}}{\left(b_{1}+c_{1}\right)_{n}\left(b_{2}+c_{2}\right)_{n} \ldots\left(b_{q}+c_{q}\right)_{n} n !}\right]=\frac{\sum_{n=0}^{\infty} A_{n}(a, b, c) x^{n}}{\sum_{n=0}^{\infty} B_{n}(a, b) x^{n}}
\end{aligned}
$$

where

$$
\begin{gathered}
A_{n}(a, b, c)=\sum_{k=0}^{n} U_{k}(a, b, c)= \\
=\sum_{k=0}^{n} \frac{\left[\left(a_{1}\right)_{k}\left(a_{1}\right)_{n-k}\right]\left[\left(a_{1}\right)_{k}\left(a_{1}\right)_{n-k}\right] \ldots\left[\left(a_{p}\right)_{k}\left(a_{p}\right)_{n-k}\right]}{\left[\left(b_{1}-c_{1}\right)_{k} \ldots\left(b_{q}-c_{q}\right)_{k}\right]\left[\left(b_{1}+c_{1}\right)_{n-k} \ldots\left(b_{q}+c_{q}\right)_{n-k}\right] k !(n-k) !},
\end{gathered}
$$

and

$$
\begin{gathered}
B_{n}(a, b)=\sum_{k=0}^{n} V_{k}(a, b)= \\
=\sum_{k=0}^{n} \frac{\left[\left(a_{1}\right)_{k}\left(a_{1}\right)_{n-k}\right]\left[\left(a_{2}\right)_{k}\left(a_{2}\right)_{n-k}\right] \ldots\left[\left(a_{p}\right)_{k}\left(a_{p}\right)_{n-k}\right]}{\left[\left(b_{1}\right)_{k}\left(b_{1}\right)_{n-k}\right]\left[\left(b_{2}\right)_{k}\left(b_{2}\right)_{n-k}\right] \ldots\left[\left(b_{q}\right)_{k}\left(b_{q}\right)_{n-k}\right] k !(n-k) !} .
\end{gathered}
$$

Now, for fixed $n \in \mathbb{N}$ we define sequences $\left(W_{n, k}(a, b, c)\right)_{k \geq 0}$ by

$W_{n, k}(a, b, c)=\frac{U_{k}(a, b, c)}{V_{k}(a, b)}=\frac{\left[\left(b_{1}\right)_{k}\left(b_{1}\right)_{n-k}\right]\left[\left(b_{2}\right)_{k}\left(b_{2}\right)_{n-k}\right] \ldots\left[\left(b_{q}\right)_{k}\left(b_{q}\right)_{n-k}\right]}{\left[\left(b_{1}-c_{1}\right)_{k} \ldots\left(b_{q}-c_{q}\right)_{k}\right]\left[\left(b_{1}+c_{1}\right)_{n-k} \ldots\left(b_{q}+c_{q}\right)_{n-k}\right]}$.

For $n, k \in \mathbb{N}$ we evaluate

$$
\begin{gathered}
\frac{W_{n, k+1}(a, b, c)}{W_{n, k}(a, b, c)}=\prod_{j=1}^{q}\left[\frac{\left(b_{j}\right)_{k+1}\left(b_{j}\right)_{n-k-1}\left(b_{j}-c_{j}\right)_{k}\left(b_{j}+c_{j}\right)_{n-k}}{\left(b_{j}\right)_{k}\left(b_{j}\right)_{n-k}\left(b_{j}-c_{j}\right)_{k+1}\left(b_{j}+c_{j}\right)_{n-k-1}}\right]= \\
=\prod_{j=1}^{q}\left[\left(\frac{\Gamma\left(b_{j}+k+1\right)}{\Gamma\left(b_{j}+k\right)}\right)\left(\frac{\Gamma\left(b_{j}+n-k-1\right)}{\Gamma\left(b_{j}+n-k\right)}\right) .\right.
\end{gathered}
$$




$$
\begin{gathered}
\left.\cdot\left(\frac{\Gamma\left(b_{j}-c_{j}+k\right)}{\Gamma\left(b_{j}-c_{j}+k+1\right)}\right)\left(\frac{\Gamma\left(b_{j}+c_{j}+n-k-1\right)}{\Gamma\left(b_{j}+c_{j}+n-k\right)}\right)\right]= \\
=\prod_{j=1}^{q}\left[\frac{b_{j}+k}{b_{j}-c_{j}+k}\right]\left[\frac{b_{j}+c_{j}+n-k-1}{b_{j}+n-k-1}\right]>1 .
\end{gathered}
$$

And now we conclude that $\left(W_{n, k}\right)_{k \geq 0}$ is increasing and consequently $\left(C_{n}=\frac{A_{n}}{B_{n}}\right)_{n \geq 0}$ is increasing too by the Lemma 1. Thus the function $x \longmapsto h_{p, q}(a ; b ; c ; x)$ is increasing on $[0,1[$ by the Lemma 2] It completes the proof of the theorem 2 .

Corollary 2 Let $p, q \in \mathbb{N}$ be such that $p \leq q+1, a=\left(a_{1}, \ldots, a_{p}\right), b=\left(b_{1}, \ldots, b_{q}\right)$, $c=\left(c_{1}, \ldots, c_{q}\right), b_{i}>0, b_{i}-c_{i}>0$ for $i=1,2, \ldots, q$ and $a_{i}>b_{i}$ for $i=2, \ldots, p$. If $b_{i}>1$ for $i=1,2, \ldots, q$, then the following Turán type inequality

$$
{ }_{p} F_{q}(a ; b-c ; x)_{p} F_{q}(a ; b+c ; x)>\left({ }_{p} F_{q}(a ; b ; x)\right)^{2}
$$

holds for all $x \in[0,1[$.

Proof Follows immediately from the monotonicity of the function $h_{p, q}(a ; b ; c ; x)$.

This Turán type inequality (10) is included as a corollary of monotonicity property we consider, this inequality itself is not new and may be found in ([12]).

There are applications of considered inequalities in the theory of transmutation operators for estimating transmutation kernels and norms $([19-[20])$ and for problems of function expansions by systems of integer shifts of Gaussians ([21]-[22]).

Recently the authors also proved generalizations of above monotonicity properties and Turán type inequalities for the case of $q$-hypergeometric functions [26]-27.

The authors are thankful to D. Karp for useful discussions.

\section{References}

[1] Sitnik S.M. Inequalities for the exponential remainder, preprint, Institute of Automation and Control Process, Far Eastern Branch of the Russian Academy of Sciences, Vladivostok, 1993 (in Russian).

[2] Edrei A., Saff E.B., Varga R.S. Zeros of Sections of Power Series. Springer, 1983.

[3] Hardy G.H., Seshu Aiyan P.V., Wilson B.M., (eds). Collected Papers of Srinivasa Ramanujan. Cambridge, 1927.

[4] Kesava Menon P. Some integral inequalities. Math. Student, 1943, Vol. 11, P. 36-38.

[5] Alzer H. An inequality for the exponential function. Arch. Math., 1990, Vol. 55, P. 462-464.

[6] Gautschi W. A note on the successive remainders of the exponential series. Elem. Math., 1982, Vol. 37, P. 46-49.

[7] Sitnik S.M., Zelezko O. Unpublished, 1994. 
[8] Sitnik S.M. A conjecture on monotonicity of a ratio of Kummer hypergeometric functions. arXiv:1207.0936, 2012, version 2, 2014. 4 pp. (http://arxiv.org/abs/1207.0936).

[9] Sitnik S.M. Conjectures on Monotonicity of Ratios of Kummer and Gauss Hypergeometric Functions. RGMIA Research Report Collection, 17(2014), Article 107, 4 pp.

[10] Karp D.B., Sitnik S.M. Log-convexity and log-concavity of hypergeometric-like functions. Journal of Mathematical Analysis and Applications, Vol. 364, No. 2, 2010, P, 384-394.

[11] Karp D.B., Sitnik S.M. Log-convexity and log-concavity of hypergeometric-like functions. arXiv:0902.3073v1, 2009.

[12] Karp D.B., Sitnik S.M. Inequalities and monotonicity of ratios for generalized hypergeometric function. Journal of Approximation Theory, 2009 , Vol. 161, P. 337-352.

[13] Kalmykov S.I., Karp D.B. Log-concavity for series in reciprocal gamma functions, Int. Transforms and Special Func. 24 (11), 859-872, 2013).

[14] Kalmykov S.I., Karp D.B. Log-convexity and log-concavity for series in gamma ratios and applications, J. Math. Anal. Appl. 406, 400-418, 2013.

[15] Karp D.B., Savenkova A., Sitnik S.M. Series expansions for the third incomplete elliptic integral via partial fraction decompositions. Journal of Computational and Applied Mathematics. Vol. 207, № 2, P. 331-337.

[16] Barnard R.W., Gordy M., Richards K.C. A note on Turán Type and Mean Inequalities for the Kummer Function. Journal of Mathematical Analysis and Applications, Vol. 349, No. 1, 2009, 259-263, doi:10.1016/j.jmaa.2008.08.024.

[17] Baricz Á. Functional inequalities involving Bessel and modified Bessel functions of the first kind. Expositiones Mathematicae, 2008, Vol. 26, P. 279 293.

[18] Lindén H. Convexity and inequalities for power series. Helsinki Analysis Seminar, 2006. 32 pp.

[19] Sitnik S.M. Factorization and Estimates of Norms in Weighted Lebesgue Spaces of Buschman-Erdelyi Operators. Soviet Math. Dokl, 1991.

[20] Katrakhov V.V., Sitnik S.M. Boundary value problem for the stationary Schroedinger equation with a singular potential. Akademiia Nauk SSSR, Doklady, 1984.

[21] Zhuravlev M.V., Kiselev E.A., Minin L.A., Sitnik S.M. Jacobi thetafunctions and systems of integral shifts of Gaussian functions. Journal of Mathematical Sciences, 2011, V. 173, No. 2, P. 231-241.

[22] Kiselev E.A., Minin L.A., Novikov I.Ya., Sitnik S.M. On the Riesz Constants for Systems of Integer Translates. Mathematical Notes, 2014, Vol. 96, No. 2, pp. 228-238. 
[23] Biernacki M., Krzyz J. On the monotonicity of certain functionals in the theory of analytic functions. Ann. Univ. M. Curie-Skłodowska, 2 (1995), $134-145$.

[24] Ponnusamy S., Vuorinen M., Asymptotic expansions and inequalities for hypergeometric functions, Mathematika 44 (1997) 278-301.

[25] Anderson G.D., Vuorinen M. Reflections on Ramanujan's Mathematical Gems. arXiv:1006.5092v1, 2010, 23 pp.

[26] K. Mehrez, S.M. Sitnik, Monotonicity of ratios of $q$-Kummer confluent hypergeometric and $q$-hypergeometric functions and associated Turán types inequalities. arXiv:1412.1634v1 [math.CA], 2014, 9 P.

[27] K. Mehrez, S.M. Sitnik, On monotonicity of ratios of $q$-Kummer confluent hypergeometric and $q$-hypergeometric functions and associated Turán types inequalities. RGMIA Research Report Collection, 17(2014), Article 150, 9 pp.

[28] K. Mehrez, S.M. Sitnik, Inequalities for Sections of Exponential Function Series and Proofs of Some Conjectures on Monotonicity of Ratios of Kummer, Gauss and Generalized Hypergeometric Functions. RGMIA Research Report Collection, 17(2014), Article 132, 8 pp.

Khaled Mehrez.

Département de Mathématiques,

l'Institut Preparatoire Aux Etudes d'Ingenieur de Monastir (IPEIM).

Monastir 5000, Tunisia.

E-mail address: k.mehrez@yahoo.fr

Sergei M. Sitnik.

Voronezh Institute of the Russian Ministry of Internal Affairs.

Voronezh, Russia.

E-mail address: pochtaname@gmail.com 